\title{
Association of Dietary Patterns with Metabolic Syndrome: Results from the Kardiovize Brno 2030 Study
}

\author{
Antonella Agodi ${ }^{1}$, Andrea Maugeri ${ }^{1,2}{ }^{(0)}$, Sarka Kunzova ${ }^{2}$, Ondrej Sochor ${ }^{2}$, Hana Bauerova ${ }^{2}$, \\ Nikola Kiacova $^{2}{ }^{(\mathbb{D}}$, Martina Barchitta ${ }^{1}$ and Manlio Vinciguerra ${ }^{2,3, *}$ \\ 1 Department of Medical and Surgical Sciences and Advanced Technologies "GF Ingrassia", University of \\ Catania, via S. Sofia 87, 95123 Catania, Italy; agodia@unict.it (A.A.); andreamaugeri88@gmail.com (A.M.); \\ martina.barchitta@unict.it (M.B.) \\ 2 International Clinical Research Center, St Anne's University Hospital, 65691 Brno, Czech Republic; \\ sarka.kunzova@fnusa.cz (S.K.); ondrej.sochor@fnusa.cz (O.S.); hana.bauerova@fnusa.cz (H.B.); \\ n.kiacova@seznam.cz (N.K.) \\ 3 Institute for Liver and Digestive Health, Division of Medicine, University College London (UCL), \\ London NW3 2PF, UK \\ * Correspondence: manlio.vinciguerra@fnusa.cz; Tel.: +420-511-158-279
}

Received: 12 June 2018; Accepted: 11 July 2018; Published: 13 July 2018

\begin{abstract}
Although metabolic syndrome (MetS) could be handled by lifestyle interventions, its relationship with dietary patterns remains unclear in populations from Central Europe. Using data from the Kardiovize Brno cohort, the present study aims to identify the main dietary patterns and to evaluate their association with MetS risk in a random urban sample from Brno, Czech Republic. In a cross-sectional study of 1934 subjects aged 25-65 years (44.3\% male), dietary patterns were derived by food frequency questionnaire (FFQ) administration and principal component analysis. Metabolic syndrome was defined according to the International Diabetes Federation statement. Logistic regression models were applied. High adherence to the prudent dietary pattern was associated with lower odds of abdominal obesity, abnormal glucose concentration, and MetS. By contrast, high adherence to the western dietary pattern was associated with higher odds of abnormal glucose, triglycerides and blood pressure levels. Whilst our results confirm the deleterious effect of a western dietary pattern on several metabolic risk factors, they also indicate that the consumption of a diet rich in cereals, fish, fruit and vegetables is associated with a healthier metabolic profile. However, further prospective research is warranted to develop and validate novel potential preventive strategies against MetS and its complications.
\end{abstract}

Keywords: diet; nutrition; metabolic disorders; obesity; hypertension; diabetes; hyperlipidemia

\section{Introduction}

Metabolic syndrome (MetS) is a growing public health concern worldwide, which is associated with an increased risk of cardiovascular morbidity and mortality [1-4]. Among the multiple classifications for the diagnosis of MetS, one of the most widely used definitions is that developed by the International Diabetes Federation (IDF) [5], which defines MetS as the combination of clinical and metabolic factors, including insulin resistance, hyperglycemia, hypertension, dyslipidemia and abdominal obesity [5]. MetS is frequently accompanied by lipid accumulation in the liver-this condition is also defined as nonalcoholic fatty liver disease (NAFLD)—which is considered the hepatic manifestation of MetS. Interestingly, NAFLD and metabolic syndrome overlap in many aspects, not only including the spectrum of diseases that they predict but also risk factors associated with them [6]. 
In the last decades, prevalence of MetS has dramatically increased, likely due to changes in lifestyle factors, socioeconomic status and dietary habits. Given this scenario, one of the greatest challenges in the management of MetS is to alleviate the risk associated with modifiable factors, such as obesity, physical activity and diet, through lifestyle interventions [7]. Although the association of dietary patterns with the risk of MetS has been well-established, as confirmed by recent meta-analyses [8,9], evidence substantially varied across populations. Whilst a "prudent" dietary pattern-a healthy diet characterized by high intakes of vegetables, fruits and fish — was inversely associated with MetS [10], a "western" dietary pattern-characterized by high intakes of red and processed meat, refined grains, alcohol and fried foods-increased the risk of MetS [11]. However, results from other studies were inconclusive $[12,13]$. This inconsistency could be attributed not only to difference in the prevalence of MetS [14], as well as in the definition used for the diagnosis [15], but also to dietary habits that vary between populations. In fact, there is considerable variation in food intakes across Europe and also within countries [16]. To explore the complex association of biological, environmental and behavioral risk factors with cardiovascular diseases (CVD), we recently designed the Kardiovize Brno 2030 study, which recruited a randomly-selected sample of residents in an urban population of Brno, Czech Republic [17]. The Kardiovize Brno 2030 study provides evidence from a European region where epidemiological studies are scarce, focusing on a population with a peculiar lifestyle: while more than half of Kardiovize subjects are highly physically active, less than five percent have a healthy diet [18]. Previous results from this population suggested that eating more frequently and consuming breakfast may be potential preventive strategies against weight gain and CVD risk [19]. In the current cross-sectional analysis, using data from the Kardiovize Brno 2030 cohort, we aim to identify the main dietary patterns and to evaluate the association of these patterns with the risk of MetS.

\section{Materials and Methods}

\subsection{Study Design}

In this cross-sectional study, we used data from the Kardiovize Brno 2030 cohort which included a random sample of residents from the city of Brno, Czech Republic [17]. The study protocol was approved by the ethics committee of St Anne's University Hospital, Brno, Czech Republic (reference 2 G/2012), in accordance with the Declaration of Helsinki. Data were collected and stored in the web-based research electronic data capture (REDCap) [20]. Demographics characteristics, socioeconomic status, cardiovascular risk behaviors and medical history were collected by trained professionals through a face-to-face comprehensive health interview. In the current analysis we included participants (i) of both sexes, (ii) aged 25-65 years and (iii) regardless menopause status in women, (iv) with no previous/current cardiovascular disease, (v) who had complete information on anthropometric measurements, cardio-metabolic parameters, sociodemographic, diet and lifestyle. We excluded subjects with extreme values of total energy intake ( $<500$ or $>3500 \mathrm{kcal})$ and diabetic patients, since the diagnosis of diabetes may influence their dietary habits.

\subsection{Dietary Assessment}

Dietary data were obtained by a 43-item Food Frequency Questionnaire (FFQ) using the previous week as reference period. The intake of alcohol drinks (i.e., wine, beer, dessert wines and spirits) was assessed using beverage-specific weekly recall. For each food item, participants were asked to indicate frequency of consumption classified into seven categories, ranging from "almost never" to "six or more times a day". Standard portion sizes-defined as the age- and sex-specific median food intake obtained from an individual dietary survey on the national level [21], which involved age and gender representative sample of the Czech population [22] —were attributed to each food item. Food intakes were derived from the FFQ by multiplying frequency of consumption by standard portion size of each food item. Food intakes were adjusted for total energy intake-assessed using the NutriDan software—by the residual method [23]. 


\subsection{Principal Component Analysis}

A posteriori dietary patterns were derived using principal component analysis (PCA). Food items were classified into 31 predefined food groups, based on the similarity of nutrient profiles or culinary usage. Individual food items that constituted a distinct item on their own (e.g., pasta, pizza, or eggs, etc.) or that represent a particular dietary pattern (e.g., alcoholic drinks and fries, etc.) were preserved. Factors analysis was performed on energy-adjusted intakes of each predefined food group followed by varimax rotation, which maintains uncorrelated factors facilitating the interpretability. The number of dietary patterns was defined based on eigenvalues $>2.0$, Scree plot examination, and interpretability. To characterize each dietary pattern we considered factor loadings with absolute value $\geq 0.250$. To confirm internal reproducibility, we separately performed factor analysis in two randomly selected subgroups by using the same abovementioned approach. For each dietary pattern, factor scores were calculated by summing the products between observed energy-adjusted food group intakes and their factor loadings. For each dietary pattern, factor scores were categorized by tertiles (T1 = low adherence; T2 = medium adherence; T3 = high adherence); the lowest tertile (T1) of each dietary pattern was used as the reference for further analyses.

\subsection{Anthropometric Measurements}

Anthropometric measurements were performed after overnight fasting by trained researchers, according to previously described protocols [17]. Briefly, height and weight were measured to the nearest $0.5 \mathrm{~cm}$ and $1 \mathrm{~kg}$, using a medical digital scale with meter (SECA 799; SECA, GmbH and Co. kg, Hamburg, Germany). BMI-defined as weight in kilograms divided by height in meters squared-was categorized as underweight $\left(<18.5 \mathrm{~kg} / \mathrm{m}^{2}\right)$, normal weight $\left(18.6-24.9 \mathrm{~kg} / \mathrm{m}^{2}\right)$, overweight (25-29.9 kg/m²), and obese $\left(\geq 30 \mathrm{~kg} / \mathrm{m}^{2}\right)$. Waist, hip and neck circumferences were measured to the nearest $1 \mathrm{~cm}$ by using a manual measuring tape. Waist-to-hip ratio (WHR) was calculated by dividing the waist measurement by hip measurement. According to ethnic specific values, abdominal obesity was defined as waist circumference $\geq 94 \mathrm{~cm}$ in men and $\geq 80 \mathrm{~cm}$ in women. Body fat mass (BFM) was assessed using a direct segmental multi-frequency bioelectrical impedance analysis (InBody 370; BIOSPACE Co., Ltd., Seoul, Korea).

\subsection{Biochemical Analyses and Physical Examination}

Cardio-metabolic parameters were assessed as described elsewhere [17]. Briefly, blood pressure (BP) was measured using a mercury sphygmomanometer (Baumanometer, W.A. Baum, Co., Inc., Copiague, NY, USA). Biochemical parameters were evaluated on 12-h fasting peripheral blood samples using a Modular SWA P800 analyzer (Roche, Basel, Switzerland). Particularly, total cholesterol, triglycerides, fasting glucose and creatinine were assayed by the enzymatic colorimetric method (Roche Diagnostics GmbH, Penzberg, Germany), while HDL- and LDL-cholesterol by the homogeneous method through direct measuring without precipitation (Sekisui Medical, Tokyo, Japan). If triglyceride levels were lower than $4.5 \mathrm{mmol} / \mathrm{L}$, we calculated LDL-cholesterol according to the Friedewald equation.

\subsection{Definition of Metabolic Syndrome}

According to the International Diabetes Federation (IDF), we defined MetS for participants with abdominal obesity who met at least two out of four criteria: fasting glucose $\geq 100 \mathrm{mg} / \mathrm{dL}(5.6 \mathrm{mmol} / \mathrm{L})$ or treatment for type 2 diabetes; HDL cholesterol $<40 \mathrm{mg} / \mathrm{dL}(1.03 \mathrm{mmol} / \mathrm{L})$ in men and $<50 \mathrm{mg} / \mathrm{dL}$ $(1.29 \mathrm{mmol} / \mathrm{L})$ in women or drug treatment for lipid abnormality; TG $\geq 150 \mathrm{mg} / \mathrm{dL}(1.7 \mathrm{mmol} / \mathrm{L})$ or drug treatment for lipid abnormality; and systolic BP $\geq 130 \mathrm{mmHg}$ or diastolic $\mathrm{BP} \geq 85 \mathrm{mmHg}$, or drug treatment for hypertension [5]. 


\subsection{Lifestyle Factors}

Physical activity level and intensity (walking, moderate, and vigorous) were assessed using the long version of the International Physical Activity Questionnaire (IPAQ-L) [24] translated in Czech, which includes four domains (leisure time, work/commuting, home and garden/yard). Physical activity was reported as Metabolic Equivalent of Task (MET-min/week) and classified as follows: high (vigorous-intensity activity on at least 3 days and accumulating at least 1500 MET-min/week or 5 or more days of any combination of walking, moderate-intensity or vigorous intensity activities achieving a minimum of at least 3000 MET-min/week); moderate (3 or more days of vigorous activity of at least $20 \mathrm{~min}$ per day or 5 or more days of moderate-intensity activity or walking of at least $30 \mathrm{~min}$ per day or 5 or more days of any combination of walking, moderate-intensity or vigorous intensity activities achieving a minimum of at least 600 MET-min/week); low (subjects who not meet criteria for categories 2 or 3) [24]. For smoking status, subjects were classified as no smokers (never being a smoker or having quit $>12$ months) and current smokers (daily or occasionally).

\subsection{Other Covariates}

Face-to-face comprehensive health interviews were carried out on demographics/socioeconomic status and medical history. The following information were recorded: demographics and socioeconomic status (age, gender, educational level, and employment status); self-reported medical history (diagnosis and treatment of hypertension and hyperlipidemia). Particularly, hypertension was defined as BP greater than or equal to $140 / 90 \mathrm{mmHg}$, or a prior diagnosis of hypertension or taking antihypertensive drugs. Hyperlipidemia was defined as having either total cholesterol greater than or equal to $5.0 \mathrm{mmol} / \mathrm{L}$, or LDL cholesterol greater than or equal to $3 \mathrm{mmol} / \mathrm{L}$, or triglycerides greater than or equal to $1.7 \mathrm{mmol} / \mathrm{L}$, or taking lipid-lowering drugs.

\subsection{Statistical Analyses}

Statistical analyses were performed using the SPSS software (version 22.0, SPSS, Chicago, IL, USA). The Kolmogorov-Smirnov test was used to assess the normal distribution of variables. Descriptive statistics were used to characterize study participants using frequencies or median and interquartile range (IQR). Trend across tertiles of dietary patterns was analyzed using Kruskal-Wallis test for continuous variables and Chi-squared test for categorical variables. To assess the association between dietary patterns and the risk of MetS and its components, multiple unconditional regression models were applied. Odds ratios (OR) and 95\% confidence intervals (CI) were calculated using the following models: model 1 adjusted for age and sex; model 2 adjusted for age, sex, marital status, employment, educational level, smoking, BMI, total energy intake, and physical activity. All statistical tests were 2 -sided, and $p$ values less than 0.05 were considered statistically significant.

\section{Results}

\subsection{Study Population and Prevalence of MetS}

A total of 1934 participants, aged between 25 and 65 years (mean $=46.6$ years in men; mean $=47.2$ years in women), were included in the current analysis. Approximately half the sample (55.7\%) was female and $23.7 \%$ were current smokers. The majority of the sample was employed $(82.0 \%)$ and married $(61.7 \%)$. Median values of total energy intake and physical activity level were $2006 \mathrm{kcal}(\mathrm{IQR}=916)$ and 3375 MET-min/week (IQR = 4782), respectively. Forty-nine \% of subjects were overweight or obese, with $52.6 \%$ meeting or exceeding the cut-offs for abdominal obesity. The prevalence of hypertension was $34.6 \%$, with $17 \%$ of participants receiving antihypertensive treatment. Specifically, median values of systolic and diastolic BP were $117.0 \mathrm{mmHg}(\mathrm{IQR}=19.5)$ and $79.0 \mathrm{mmHg}(\mathrm{IQR}=13.0)$, respectively. The prevalence of hyperlipidemia was $65.4 \%$, with $5.4 \%$ receiving drug treatment for lipid abnormality. Specifically, median values of HDL and triglycerides were $1.52(\mathrm{IQR}=0.50)$ and $0.99(\mathrm{IQR}=0.76)$, respectively. Median fasting glucose level was $4.8(\mathrm{IQR}=0.7)$, and none of the participants received 
antidiabetic treatment since we excluded diabetic patients from the current analysis. Based on the IDF definition, prevalence of MetS was 38.2\%, with $41.0 \%, 15.9 \%, 22.0 \%$ and $9.5 \%$ of subjects who showed abnormal BP, HDL, triglycerides and fasting glucose, respectively.

Table 1 shows the comparison of socio-demographic characteristics, anthropometric measures and cardio-metabolic parameters between MetS patients and controls. Briefly, MetS group consisted of more male $(48.6 \%$ vs. $42.2 \% ; p=0.017)$ and older subjects (median $=54.0$ years, IQR $=15.0$ vs. median $=41.5$ years, IQR $=17.0 ; p<0.001)$, with higher percentages of low-educated $(61.0 \%$ vs. $46.4 \%$; $p<0.001)$, married (69.1\% vs. $58.3 \%$; $p<0.001)$, unemployed $(25.7 \%$ vs. $13.3 \% ; p<0.001)$ and smoking (26.7\% vs. $22.1 \% ; p=0.041)$ subjects. As expected, MetS patients exhibited higher anthropometric measures and cardio-metabolic parameters, but lower total energy intake, physical activity and HDL cholesterol level than controls.

Table 1. Characteristics of study population and comparison between MetS patients and controls.

\begin{tabular}{|c|c|c|c|c|}
\hline Characteristics & $\begin{array}{c}\text { Total } \\
(N=1934)\end{array}$ & $\begin{array}{c}\text { MetS } \\
(N=739)\end{array}$ & $\begin{array}{c}\text { Control } \\
(N=1195)\end{array}$ & $p$-Value ${ }^{a}$ \\
\hline Age, years $b$ & $47.0(19.0)$ & $54.0(15.0)$ & $41.5(17.0)$ & $<0.001$ \\
\hline Sex (\% male $)$ & $44.6 \%$ & $48.6 \%$ & $42.2 \%$ & 0.017 \\
\hline Marital status ( $\%$ married) & $61.7 \%$ & $69.1 \%$ & $58.3 \%$ & $<0001$ \\
\hline Employment (\% unemployed) & $18.1 \%$ & $25.7 \%$ & $13.3 \%$ & $<0.001$ \\
\hline Smoking (\% current smokers) & $23.7 \%$ & $26.7 \%$ & $22.1 \%$ & 0.041 \\
\hline Total energy intake, $\mathrm{kcal}^{\mathrm{b}}$ & $2006(916)$ & 1963 (912) & $2045(910)$ & 0.013 \\
\hline Physical activity, MET min/week ${ }^{b}$ & $3175(4782)$ & $2994(4388)$ & $3252(4340)$ & 0.009 \\
\hline Hypertension & $34.6 \%$ & $66.0 \%$ & $18.2 \%$ & $<0.001$ \\
\hline Use of antihypertensives & $17.0 \%$ & $36.5 \%$ & $5.2 \%$ & $<0.001$ \\
\hline Hyperlipidemia & $65.4 \%$ & $84.3 \%$ & 54.0 & $<0.001$ \\
\hline Use of hypolipidemics & $5.4 \%$ & $11.2 \%$ & $1.9 \%$ & $<0.001$ \\
\hline Weight, kg ${ }^{\mathrm{b}}$ & $75.7(23.0)$ & $88.3(20.2)$ & $69.8(17.9)$ & \\
\hline $\mathrm{BMI}, \mathrm{kg} / \mathrm{m}^{2 \mathrm{~b}}$ & $24.8(5.8)$ & $28.6(5.3)$ & $23.1(3.9)$ & $<0.001$ \\
\hline \multicolumn{5}{|l|}{ BMI categories (\%) } \\
\hline Underweight & $2.9 \%$ & $0 \%$ & $4.6 \%$ & \multirow{4}{*}{$<0.001$} \\
\hline Normal weight & $48.5 \%$ & $14.7 \%$ & $69.3 \%$ & \\
\hline Overweight & $33.6 \%$ & $50.5 \%$ & $23.1 \%$ & \\
\hline Obese & $15.1 \%$ & $34.8 \%$ & $3.0 \%$ & \\
\hline Waist circumference ${ }^{b}$ & $87.0(20.0)$ & $100.0(13.0)$ & $81.0(14.0)$ & $<0.001$ \\
\hline Hip circumference ${ }^{b}$ & $102.0(10.0)$ & $107.0(10.0)$ & $99.0(8.0)$ & $<0.001$ \\
\hline WHR ${ }^{b}$ & $0.85(0.14)$ & $0.92(0.12)$ & $0.81(0.12)$ & $<0.001$ \\
\hline Abdominal Obesity (\%) & $52.2 \%$ & $100 \%$ & $22.6 \%$ & $<0.001$ \\
\hline Body Fat Mass b & $18.4(12.1)$ & $25.5(11.1)$ & $14.6(8.0)$ & $<0.001$ \\
\hline Systolic Blood Pressure, $\mathrm{mmHg}$ & $117.0(19.5)$ & $127.5(19.8)$ & $112.5(14.0)$ & $<0.001$ \\
\hline Diastolic Blood Pressure, $\mathrm{mmHg}$ & $79.0(13.0)$ & $85.5(11.3)$ & $76.3(10.5)$ & $<0.001$ \\
\hline Glycated Haemoglobin, $\mathrm{nmol} / \mathrm{mol}^{\mathrm{b}}$ & $39.0(6.0)$ & $41.0(5.0)$ & $38.0(5.0)$ & $<0.001$ \\
\hline Fasting Glucose, $\mathrm{nmol} / \mathrm{L}^{\mathrm{b}}$ & $4.8(0.7)$ & $5.1(0.7)$ & $4.7(0.6)$ & $<0.001$ \\
\hline Creatinine, $\mathrm{nmol} / \mathrm{L}^{\mathrm{b}}$ & $75.0(18.0)$ & $76.0(18.5)$ & $75.0(18.0)$ & 0.312 \\
\hline Triglycerides, $\mathrm{nmol} / \mathrm{L}^{\mathrm{b}}$ & $1.0(0.8)$ & $1.4(0.9)$ & $0.8(0.5)$ & $<0.001$ \\
\hline Total Cholesterol, $\mathrm{nmol} / \mathrm{L}^{\mathrm{b}}$ & $5.1(1.3)$ & $5.4(1.4)$ & $4.9(1.3)$ & $<0.001$ \\
\hline HDL Cholesterol, $\mathrm{nmol} / \mathrm{L}^{\mathrm{b}}$ & $1.5(0.5)$ & $1.3(0.5)$ & $1.6(0.5)$ & $<0.001$ \\
\hline LDL Cholesterol, $\mathrm{nmol} / \mathrm{L}^{\mathrm{b}}$ & $3.0(1.2)$ & $3.3(1.3)$ & $2.9(1.2)$ & $<0.001$ \\
\hline Total Cholesterol/HDL-ratio ${ }^{b}$ & $3.3(1.4)$ & $4.0(1.5)$ & $3.0(1.1)$ & $<0.001$ \\
\hline
\end{tabular}

Abbreviations: IQR Interquartile range; MET Metabolic Equivalent of Task; BMI body mass index; WHR waist-to-hip ratio. ${ }^{a}$ Statistically significant $p$-values $(p<0.05)$ are indicated in bold font. ${ }^{\mathrm{b}}$ Data reported as median (IQR). 


\subsection{Dietary Patterns}

Based on the Scree plot (Figure 1), we derived two major dietary patterns with eigenvalues $\geq 2.0$ explaining 13.73\% of total variance among 31 food groups. Figures 2 and 3 illustrate factor loadings which characterized each dietary pattern. Accordingly, the first dietary pattern-named western-was positively characterized by high intake of white bread, processed meat, fries, hamburger, hot-dog and salty snacks. By contrast, the second one-named prudent according to the well-accepted term used in this field of research-was positively characterized by high intake of cereals, jam and honey, fish, fruit, raw and cooked vegetables and nuts. Characteristics of study participants by dietary pattern tertiles are reported in Table 2. Particularly, higher adherence to the western dietary pattern was associated with decreasing age and percentage of unemployed, as well as with increasing total energy intake and percentage of male. No statistically significant differences were observed across tertiles of prudent dietary pattern.

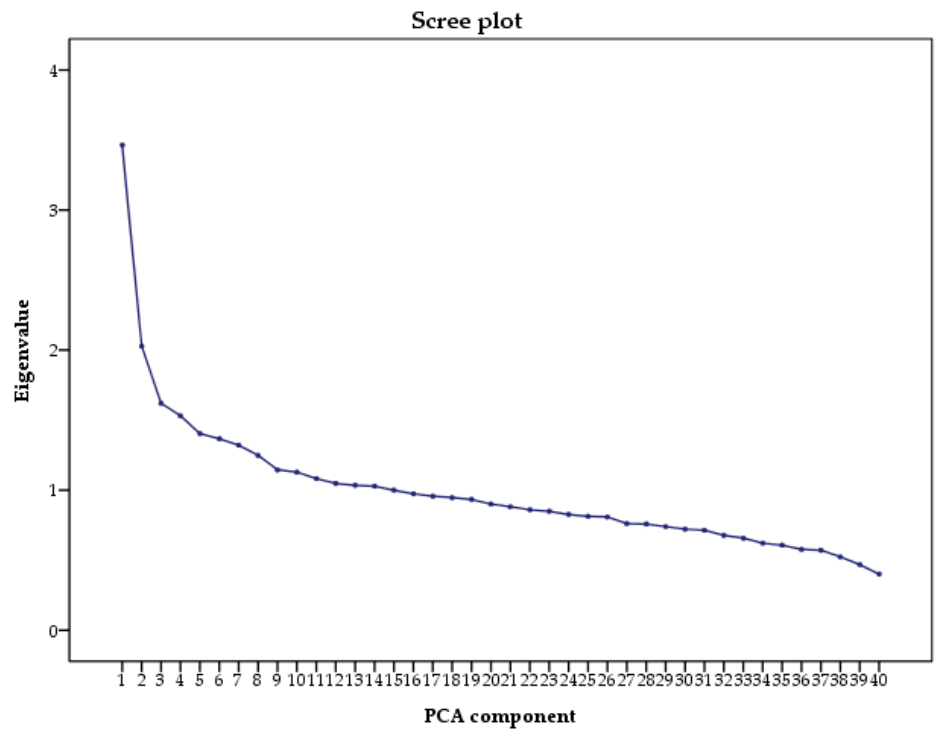

Figure 1. Scree plot of the eigenvalues. We used the scree plot examination to determine the appropriate number of dietary pattern. Scree plot represents the partitioning of the total variation (i.e., eigenvalue) accounted for each principal component, against the principal component number. PCA: principal component analysis. 


\begin{tabular}{|c|c|c|}
\hline & Western & Prudent \\
\hline White bread & 0.485 & -0.238 \\
\hline Wholemeal bread & -0.194 & 0.202 \\
\hline Cereals & -0.155 & 0.369 \\
\hline Butter & -0.060 & 0.026 \\
\hline Margarine & 0.094 & -0.016 \\
\hline Milk & 0.099 & 0.092 \\
\hline Yogurt & -0.119 & 0.182 \\
\hline $\mathrm{D}$ airy D essert & 0.019 & 0.234 \\
\hline Soft cheese & 0.081 & 0.028 \\
\hline Hard cheese & -0.011 & 0.130 \\
\hline Jam and honey & -0.122 & 0.251 \\
\hline Egg & 0.127 & -0.102 \\
\hline Processed meat & 0.607 & -0.169 \\
\hline Red meat & 0.067 & -0.169 \\
\hline White meat & 0.006 & 0.016 \\
\hline Fish & -0.003 & 0.308 \\
\hline Soy products & -0.108 & -0.068 \\
\hline Fruit & -0.049 & 0.637 \\
\hline Raw vegetable & -0.065 & 0.369 \\
\hline Cooked vegetable & -0.069 & 0.266 \\
\hline Legumes & 0.033 & 0.143 \\
\hline Fries & 0.277 & -0.163 \\
\hline Rice & -0.182 & -0.055 \\
\hline Pasta & 0.020 & 0.025 \\
\hline Dumplings & 0.110 & -0.015 \\
\hline Pizza & 0.216 & -0.111 \\
\hline Hamburger and hot dog & 0.405 & -0.252 \\
\hline Nuts & -0.119 & 0.446 \\
\hline Sweets & 0.161 & 0.060 \\
\hline Salty snacks & 0.633 & 0.182 \\
\hline Alcoholic drinks & 0.066 & -0.038 \\
\hline-1 & \multicolumn{2}{|c|}{ Factor loadings } \\
\hline
\end{tabular}

Figure 2. Table of factor loadings characterizing each dietary pattern. Food groups that negatively characterize the dietary patterns are indicated in red. Food groups that positively characterize the dietary patterns are indicated in green. Factor loadings $\geq|0.25|$ are in bold font. 


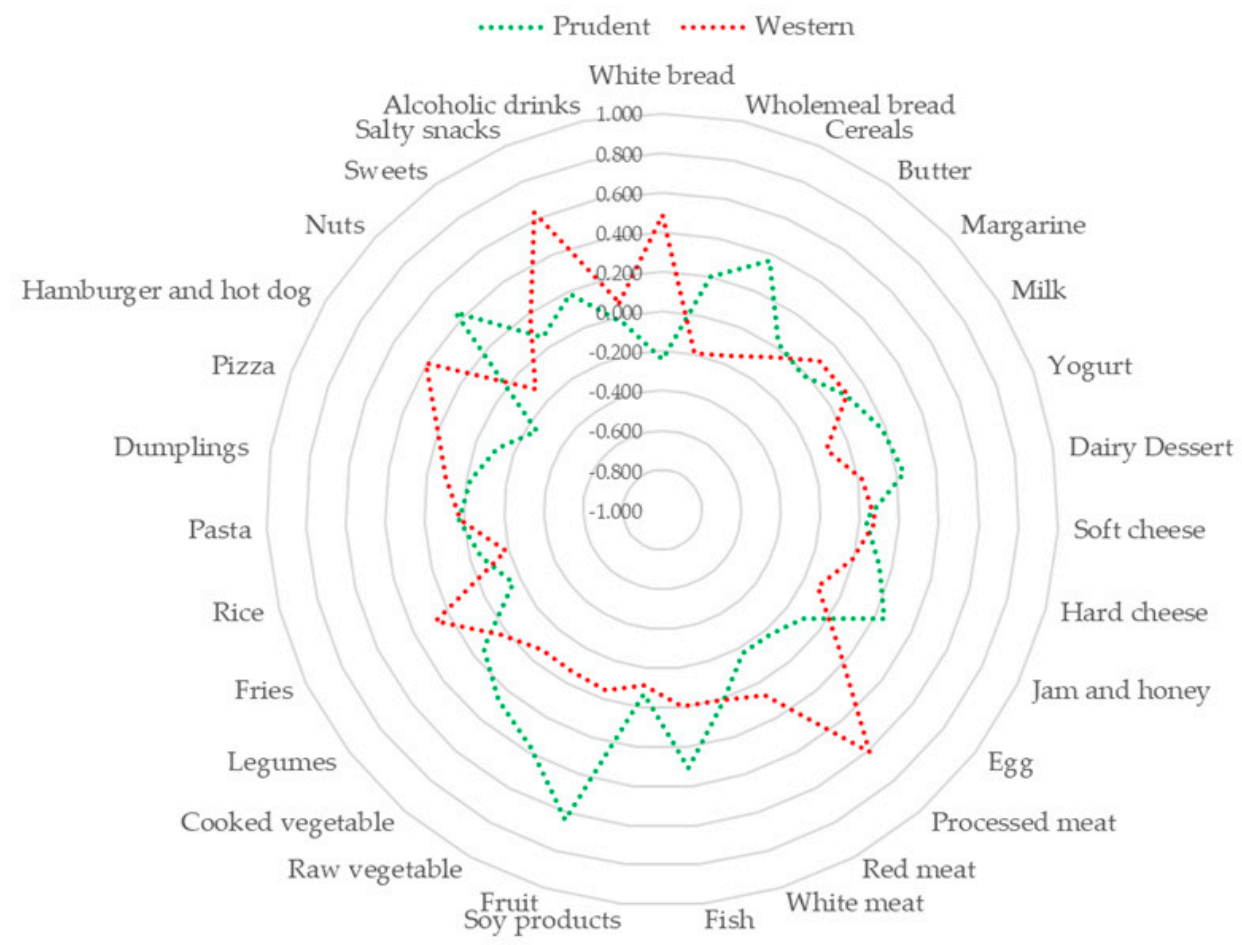

Figure 3. Radar graph of factor loadings characterizing each dietary pattern. Red line indicates factor loadings related to the western dietary pattern. Green line indicates the factor loadings related to the prudent dietary pattern.

Table 2. Characteristics of study participants by tertiles of dietary patterns.

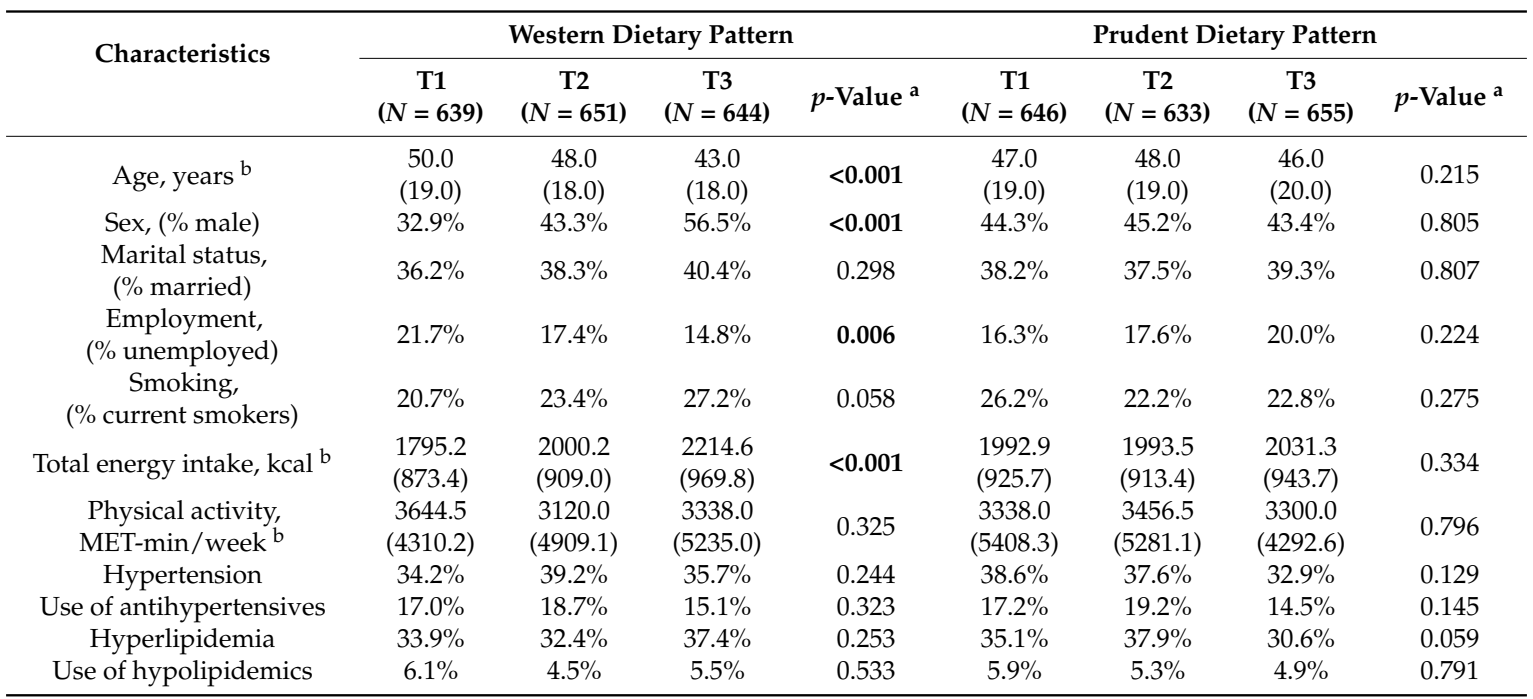

Abbreviations: T tertile; IQR Interquartile range; MET Metabolic Equivalent of Task. ${ }^{\text {a }}$ Statistically significant $p$-values $(p<0.05)$ are indicated in bold font. ${ }^{\mathrm{b}}$ Data reported as median (IQR).

\subsection{The Association of Dietary Patterns with Cardio-Metabolic Parameters}

Table 3 shows cardio-metabolic parameters by tertiles of dietary patterns. From T1 to T3 of western dietary pattern, we observed increasing weight, waist circumference, WHR, creatinine, triglycerides and total cholesterol/HDL cholesterol ratio increased, while glycated hemoglobin and HDL cholesterol decreased ( $p$-values $<0.05$ ). By contrast, from T1 to T3 of prudent dietary pattern, we observed decreasing weight, BMI, body fat mass, waist and hip circumferences, as well as the prevalence of 
overweight/obese and abdominal obese subjects ( $p$-values $<0.05$ ). In addition, systolic and diastolic BP decreased with increasing tertiles of prudent dietary pattern ( $p$-values $<0.05)$.

Table 3. Cardio-metabolic parameters of study participants by tertiles of dietary patterns.

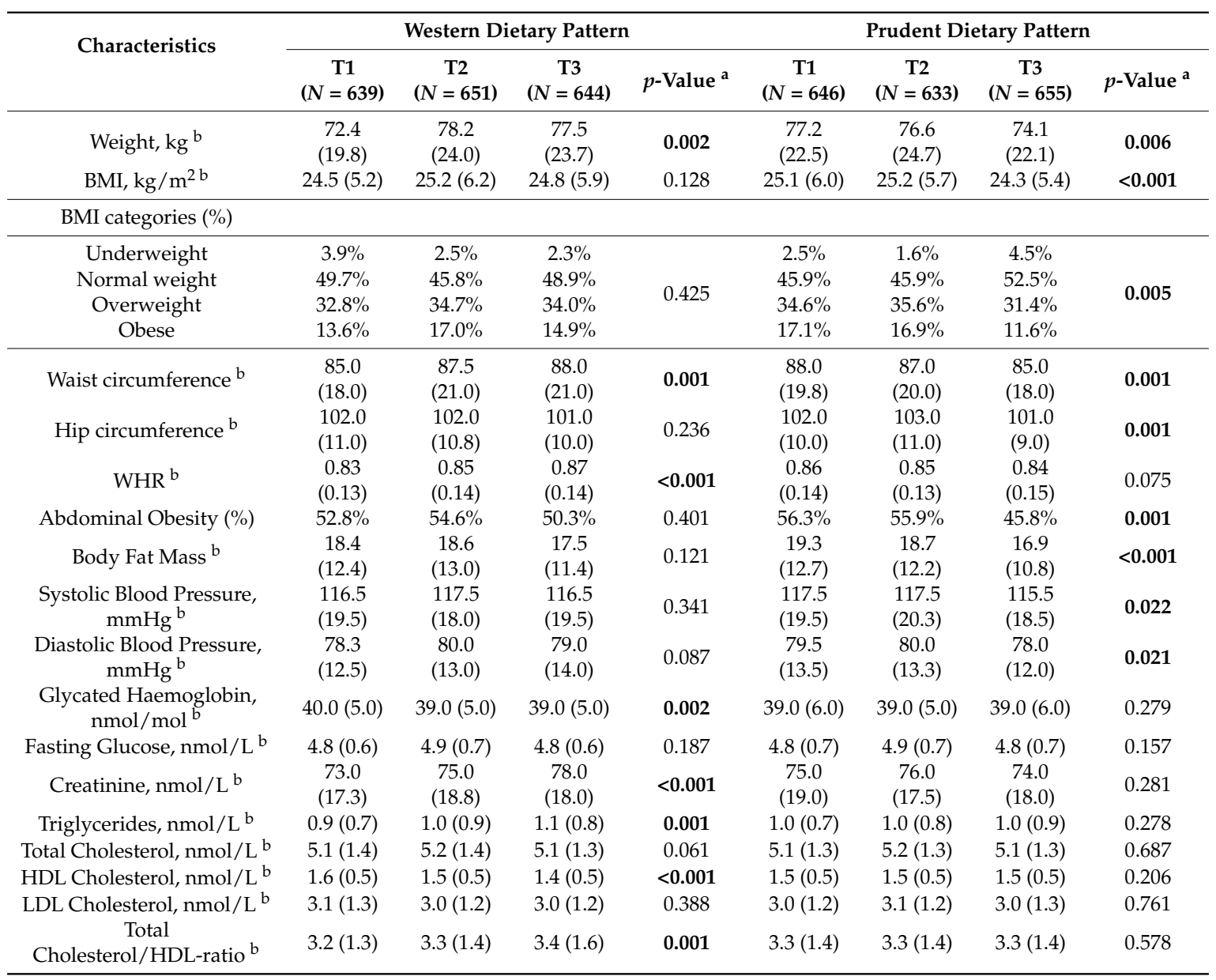

Abbreviations: T tertile; IQR Interquartile range. BMI body mass index; WHR waist-to-hip ratio. ${ }^{\text {a }}$ Statistically significant $p$-values $(p<0.05)$ are indicated in bold font. ${ }^{b}$ Data reported as median (IQR).

\subsection{The Association of Dietary Patterns with MetS and Its Components}

Figure 4 shows results from the logistic regression analysis of the association between western dietary pattern, MetS and its components. After adjustment for age and sex, higher factor score of western dietary pattern was associated with increased odds of abnormal BP, triglycerides and fasting glucose ( $p$-trend $=0.009 ; p$-trend $=0.005 ; p$-trend $=0.015$, respectively). The age- and sex-adjusted model also demonstrated that subjects in T2 and in T3 had higher odds of abnormal BP (OR $=1.55$, $95 \% \mathrm{CI}=1.17-2.05, p=0.002 ; \mathrm{OR}=1.40,95 \% \mathrm{CI}=1.04-1.88, p=0.026$; respectively), triglycerides $(\mathrm{OR}=1.44,95 \% \mathrm{CI}=1.04-2.00, p=0.029 ; \mathrm{OR}=1.64,95 \% \mathrm{CI}=1.17-2.31, p=0.005$; respectively $)$ and fasting glucose $(\mathrm{OR}=1.72,95 \% \mathrm{CI}=1.07-2.78, p=0.026 ; \mathrm{OR}=1.72,95 \% \mathrm{CI}=1.07-2.80, p=0.028$; respectively), than those in T1. Subjects in T3 also showed higher odds of abnormal triglycerides $(\mathrm{OR}=1.56,95 \% \mathrm{CI}=1.02-2.39, p=0.003)$, after adjusting for age, sex, marital status, employment, educational level, smoking, BMI, total energy intake, and physical activity. 


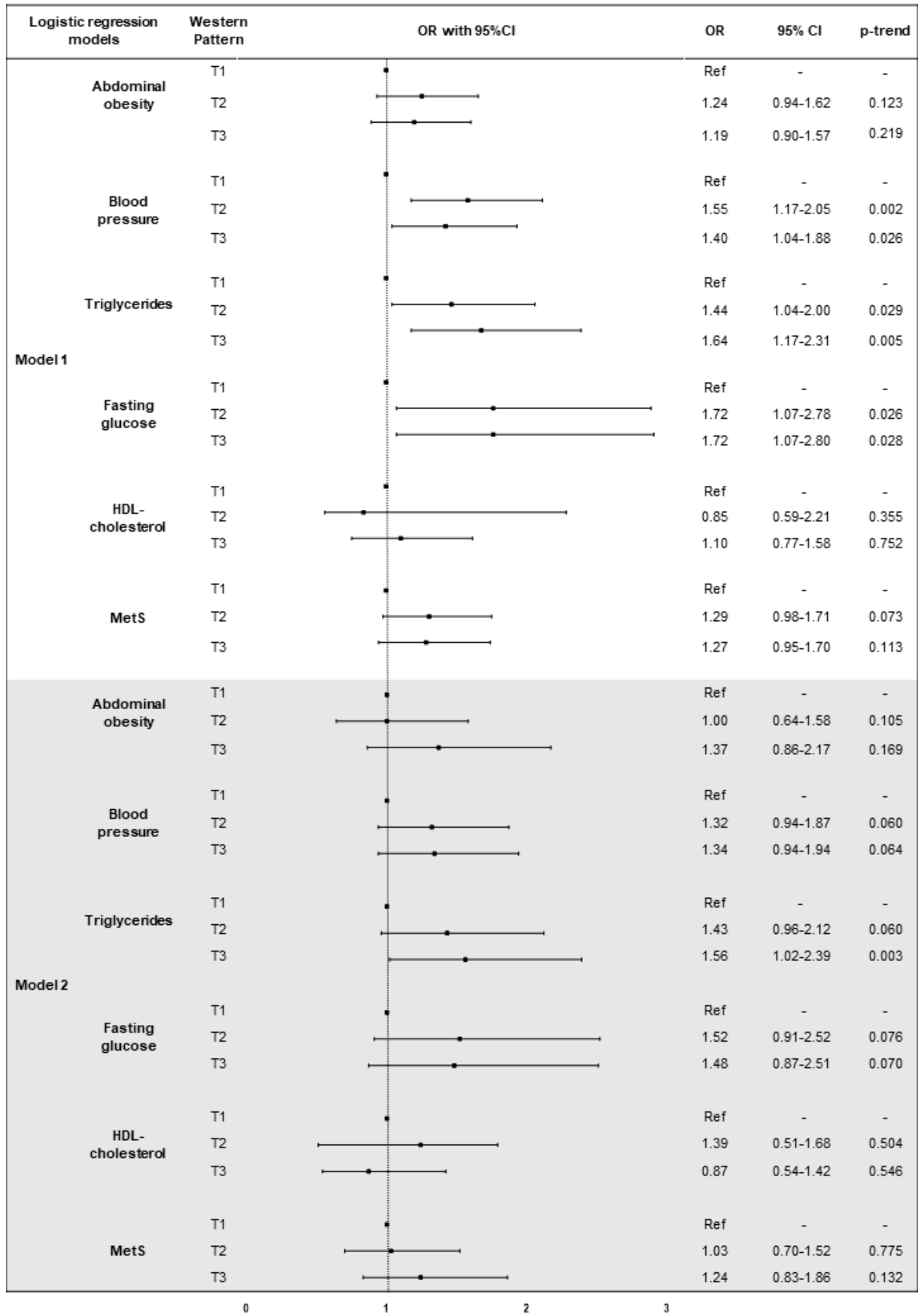

Figure 4. Logistic regression models of the association between western dietary pattern, MetS and it components. Abbreviations: $\mathrm{OR}$, odds ratio; $\mathrm{CI}$, confidence interval; T, tertile; MetS, metabolic syndrome. Model 1: adjusted for age and sex. Model 2: adjusted for age, sex, marital status, employment, educational level, smoking, BMI, total energy intake, and physical activity.

Figure 5 shows results from the logistic regression analysis of the association between prudent dietary pattern, MetS and its components. After adjustment for age and sex, higher factor score of prudent dietary pattern was associated with lower odds of abdominal obesity, abnormal BP and MetS ( $p$-trend $=0.001 ; p$-trend $=0.048 ; p$-trend $=0.003$, respectively). The age- and sex-adjusted model also showed that subjects in T3 had lower odds of abdominal obesity $(\mathrm{OR}=0.63,95 \% \mathrm{CI}=0.48-0.83$, $p=0.001)$, abnormal BP (OR $=0.76,95 \% \mathrm{CI}=0.57-0.99, p=0.049)$, fasting glucose ( $\mathrm{OR}=0.60$, $95 \% \mathrm{CI}=0.39-0.96, p=0.035)$, and MetS $(\mathrm{OR}=0.65,95 \% \mathrm{CI}=0.49-0.86, p=0.003)$, than those in 
T1. In addition, we confirmed that subjects in T3 had lower odds of abdominal obesity $(\mathrm{OR}=0.63$, $95 \% \mathrm{CI}=0.48-0.84, p=0.001)$, abnormal fasting glucose $(\mathrm{OR}=0.58,95 \% \mathrm{CI}=0.35-0.94, p=0.039)$, and MetS $(\mathrm{OR}=0.65,95 \% \mathrm{CI}=0.47-0.88, p=0.004)$, after adjusting for age, sex, marital status, employment, educational level, smoking, BMI, total energy intake, and physical activity.

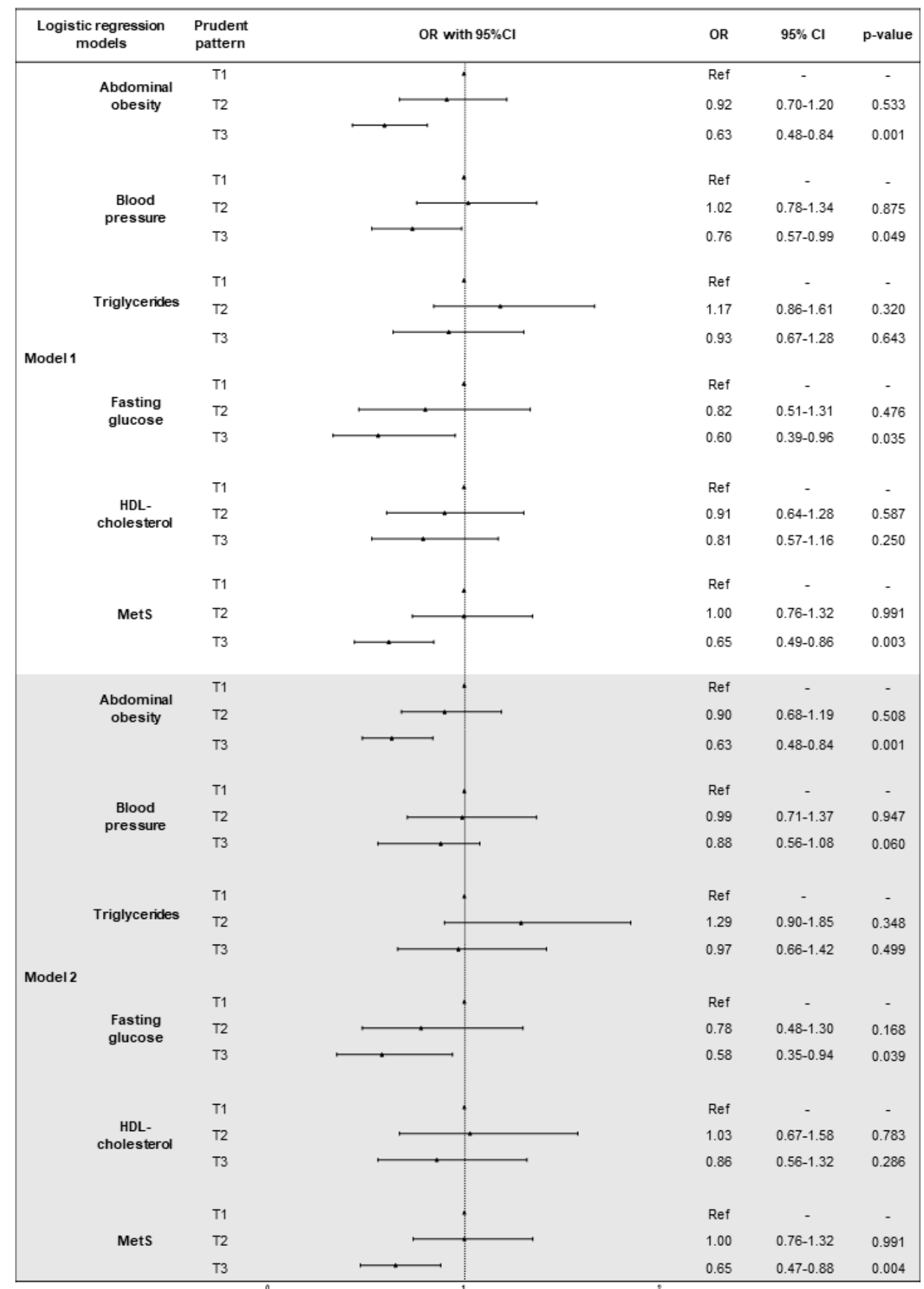

Figure 5. Logistic regression models of the association between prudent dietary pattern, MetS and it components. Abbreviations: OR, odds ratio; CI, confidence interval; T, tertile; MetS, metabolic syndrome. Model 1: adjusted for age and sex. Model 2: adjusted for age, sex, marital status, employment, educational level, smoking, BMI, total energy intake, and physical activity. 


\section{Discussion}

In the current study, according to the IDF definition [5], we reported that prevalence of MetS was $38.2 \%$ in a randomly-selected sample of the Czech urban population, which is similar with that reported among Polish, neighboring Central European population [25]. The diagnosis of MetS was more common among male, low-educated, unemployed and smoking subjects. Moreover, it was observed higher prevalence of MetS with decreasing physical activity level. To evaluate the association with MetS, we first derived two a posteriori dietary patterns which characterized the dietary habits of the study population. The first pattern—named "western" —was characterized by high intake of white bread, processed meat, fries, hamburger, hot-dog and salty snacks; the second one-named "prudent" — consisted of high intake of cereals, jam and honey, fish, fruit, raw and cooked vegetables, and nuts. Foods composition of these dietary patterns partially overlapped with those examined by two previous meta-analyses [8,9].

Consistently with results from the Polish population [25], we demonstrated the association of western dietary pattern with some of the individual MetS components, but not with overall MetS risk. Although a previous meta-analysis, comparing the highest to the lowest categories of western/unhealthy dietary patterns, reported a pooled OR for MetS of 1.28 (95\%CI = 1.17-1.40), results varied across studies [8]. This inconsistency could be attributed to differences in socio-demographic characteristics, ethnicity, behavioral and lifestyle factors. In the present study we observed that weight, waist circumference and WHR increased with increasing adherence to western dietary pattern, but we failed to replicate previous evidence showing that unhealthy dietary habits may increase the risk of abdominal obesity [26-29]. Compared to low adherence, medium-high adherence to the western dietary pattern was associated with increased odds of abnormal BP, triglycerides and fasting glucose, after adjusting for age and sex. This is consistent with several lines of evidence demonstrating that western dietary patterns were associated with levels of blood pressure and serum lipids [30-32]. By contrast, other studies reported that dietary habits, characterized by high consumption of meat, affected HDL levels but not blood pressure, LDL and triglycerides levels [25,33]. However, we confirmed a robust positive association between adherence to western dietary pattern and abnormal triglycerides $(\mathrm{OR}=1.56,95 \% \mathrm{CI}=1.02-2.39)$, by adding to the regression model both socio-demographic (i.e., marital status, employment and educational level) and behavioral factors (i.e., smoking, BMI, total energy intake and physical activity). Since MetS and NAFLD predict the same metabolic risk profile, then it follows that the two disorders should share similar pathophysiological features [6]. In fact, a similar dietary pattern rich in animal food was also associated with the risk of fatty liver disease in general [34], and NAFLD in particular [35].

By contrast, we demonstrated lower odds of MetS (OR $=0.65,95 \% C I=0.47-0.88)$ among participants with high adherence to prudent dietary pattern, after adjusting for age, sex, marital status, employment, educational level, smoking, BMI, total energy intake, and physical activity. This is consistent with results from the abovementioned meta-analysis showing a pooled OR of 0.83 $(95 \% \mathrm{CI}=0.76-0.90)$ [8]. Accordingly, our results reported decreasing blood pressure and prevalence of abdominal obesity with increasing adherence to prudent dietary pattern. In fact, the age- and sex-adjusted model showed that subjects with high adherence had lower odds of abdominal obesity $(\mathrm{OR}=0.63,95 \% \mathrm{CI}=0.48-0.83)$, as well as abnormal $\mathrm{BP}(\mathrm{OR}=0.76,95 \% \mathrm{CI}=0.57-0.99)$ and fasting glucose $(\mathrm{OR}=0.60,95 \% \mathrm{CI}=0.39-0.96)$, than those with low adherence. In addition, we established a robust association of adherence to prudent dietary pattern with abdominal obesity $(\mathrm{OR}=0.63$, $95 \% \mathrm{CI}=0.48-0.84)$ and abnormal fasting glucose $(\mathrm{OR}=0.58,95 \% \mathrm{CI}=0.35-0.94)$, by adding to the regression model both socio-demographic (i.e., marital status, employment and educational level) and behavioral factors (i.e., smoking, BMI, total energy intake and physical activity). Our results are in line with previous studies demonstrating the protective effect of healthy dietary patterns against the risk of central obesity [27,36-38], impaired fasting glucose [39], insulin resistance [40], and diabetes [41]. All these pathological features are also related to NAFLD and it has been similarly proved that adherence to a prudent dietary pattern reduced the risk of NAFLD [42]. 
A weakness of our study includes the cross-sectional design, which does not allow demonstrating the causality of the relationships. In fact, it is possible that the diagnosis of metabolic disorders may influence dietary habits. Moreover, the effect of unmeasured socio-demographic factors (e.g., income, food security, food access and food availability) physiological condition (e.g., menopause status) and comorbidities cannot be completely excluded. We estimated food intakes using a FFQ with standard portion sizes, which does not preclude measurement error and may suffer from inaccuracies. Nevertheless, standard portion sizes were obtained from an individual dietary survey on the national level, which involved age and gender representative sample of the Czech population [22]. Accordingly, the food compositions of our PCA-derived dietary patterns were consistent with those identified by previous studies across different European populations [11,25,28,43,44].

Our study has also several strengths, including the large sample that was randomly selected from the urban population of Brno (Czech Republic). Therefore, these results, obtained from a nationally representative sample, can be extrapolated to the general Czech population. Moreover, cardio-metabolic parameters and anthropometric measures were assessed using standard and validated protocols, allowing comparisons with other well-designed studies. To this purpose, we classified study participants according to the IDF definition [5], which provides one of the most widely used classifications of MetS. Finally, the majority of our results are robust, since they have been confirmed after adjusting for several socio-demographic and behavioral factors.

\section{Conclusions}

To our knowledge, the present study is the first investigating the association of dietary patterns with MetS in a random large sample of Czech urban population. Whilst we confirmed the deleterious effect of a western dietary pattern on several metabolic risk factors, our results also indicated that the consumption of a diet rich in cereals, fish, fruit and vegetables is associated with a healthier metabolic profile and lower risk of MetS. Although additional prospective research should be conducted, public health professionals could benefit from these findings, developing and validating novel potential preventive strategies against MetS and its complications.

Author Contributions: Conceptualization, A.A., A.M. and M.V.; Methodology, H.B. and N.K.; Formal Analysis, A.M. and M.B.; Resources, M.V.; Data Curation, S.K.; Writing-Original Draft Preparation, A.A., A.M., M.B. and M.V.; Writing-Review and Editing, A.A., A.M., M.B., O.S. and M.V.; Supervision, M.V.; Project Administration, M.V.; Funding Acquisition, M.V.

Funding: This research was funded by project no. LQ1605 from the National Program of Sustainability II (MEYS CR). Funding was used for data collection. A.A., A.M. and M.B. are supported by the Department of Medical and Surgical Sciences and Advanced Technologies "GF Ingrassia", University of Catania (Piano triennale di sviluppo delle attività di ricerca scientifica del Dipartimento-2016-18).

Acknowledgments: We are grateful to Jindrich Fiala for his help and expertise on nutritional assessment.

Conflicts of Interest: The authors declare no conflict of interest.

\section{References}

1. Lakka, H.M.; Laaksonen, D.E.; Lakka, T.A.; Niskanen, L.K.; Kumpusalo, E.; Tuomilehto, J.; Salonen, J.T. The metabolic syndrome and total and cardiovascular disease mortality in middle-aged men. JAMA 2002, 288, 2709-2716. [CrossRef] [PubMed]

2. Wang, J.; Ruotsalainen, S.; Moilanen, L.; Lepistö, P.; Laakso, M.; Kuusisto, J. The metabolic syndrome predicts cardiovascular mortality: A 13-year follow-up study in elderly non-diabetic Finns. Eur. Heart J. 2007, 28, 857-864. [CrossRef] [PubMed]

3. Aggarwal, A.; Aggarwal, S.; Sharma, V. Cardiovascular risk factors in young patients of coronary artery disease: Differences over a decade. J. Cardiovasc. Thorac. Res. 2014, 6, 169-173. [CrossRef] [PubMed]

4. Farhangi, M.A.; Jahangiry, L.; Asghari-Jafarabadi, M.; Najafi, M. Association between dietary patterns and metabolic syndrome in a sample of Tehranian adults. Obes. Res. Clin. Pract. 2016, 10 (Suppl. 1), S64-S73. [CrossRef] [PubMed] 
5. Alberti, K.G.; Eckel, R.H.; Grundy, S.M.; Zimmet, P.Z.; Cleeman, J.I.; Donato, K.A.; Fruchart, J.C.; James, W.P.; Loria, C.M.; Smith, S.C.; et al. Harmonizing the metabolic syndrome: A joint interim statement of the international diabetes federation task force on epidemiology and prevention; national heart, lung, and blood institute; american heart association; world heart federation; international atherosclerosis society; and international association for the study of obesity. Circulation 2009, 120, 1640-1645. [PubMed]

6. Yki-Järvinen, H. Non-alcoholic fatty liver disease as a cause and a consequence of metabolic syndrome. Lancet Diabetes Endocrinol. 2014, 2, 901-910. [CrossRef]

7. Grundy, S.M.; Cleeman, J.I.; Daniels, S.R.; Donato, K.A.; Eckel, R.H.; Franklin, B.A.; Gordon, D.J.; Krauss, R.M.; Savage, P.J.; Smith, S.C.; et al. Diagnosis and management of the metabolic syndrome. An American heart association/national heart, lung, and blood institute scientific statement. Executive summary. Cardiol. Rev. 2005, 13, 322-327. [CrossRef] [PubMed]

8. Rodríguez-Monforte, M.; Sánchez, E.; Barrio, F.; Costa, B.; Flores-Mateo, G. Metabolic syndrome and dietary patterns: A systematic review and meta-analysis of observational studies. Eur. J. Nutr. 2017, 56, 925-947. [CrossRef] [PubMed]

9. Shab-Bidar, S.; Golzarand, M.; Hajimohammadi, M.; Mansouri, S. A posteriori dietary patterns and metabolic syndrome in adults: A systematic review and meta-analysis of observational studies. Public Health Nutr. 2018, 21, 1681-1692. [CrossRef] [PubMed]

10. Esmaillzadeh, A.; Kimiagar, M.; Mehrabi, Y.; Azadbakht, L.; Hu, F.B.; Willett, W.C. Dietary patterns, insulin resistance, and prevalence of the metabolic syndrome in women. Am. J. Clin. Nutr. 2007, 85, 910-918. [CrossRef] [PubMed]

11. Heidemann, C.; Scheidt-Nave, C.; Richter, A.; Mensink, G.B. Dietary patterns are associated with cardiometabolic risk factors in a representative study population of German adults. Br. J. Nutr. 2011, 106, 1253-1262. [CrossRef] [PubMed]

12. Hong, S.; Song, Y.; Lee, K.H.; Lee, H.S.; Lee, M.; Jee, S.H.; Joung, H. A fruit and dairy dietary pattern is associated with a reduced risk of metabolic syndrome. Metabolism 2012, 61, 883-890. [CrossRef] [PubMed]

13. Cho, Y.A.; Kim, J.; Cho, E.R.; Shin, A. Dietary patterns and the prevalence of metabolic syndrome in Korean women. Nutr. Metab. Cardiovasc. Dis. 2011, 21, 893-900. [CrossRef] [PubMed]

14. Cameron, A.J.; Shaw, J.E.; Zimmet, P.Z. The metabolic syndrome: Prevalence in worldwide populations. Endocrinol. Metab. Clin. N. Am 2004, 33, 351-375. [CrossRef] [PubMed]

15. Day, C. Metabolic syndrome, or what you will: Definitions and epidemiology. Diabetes Vasc. Dis. Res. 2007, 4, 32-38. [CrossRef] [PubMed]

16. Mertens, E.; Kuijsten, A.; Dofková, M.; Mistura, L.; D’Addezio, L.; Turrini, A.; Dubuisson, C.; Favret, S.; Havard, S.; Trolle, E.; et al. Geographic and socioeconomic diversity of food and nutrient intakes: A comparison of four European countries. Eur. J. Nutr. 2018. [CrossRef] [PubMed]

17. Movsisyan, N.K.; Vinciguerra, M.; Lopez-Jimenez, F.; Kunzová, Š.; Homolka, M.; Jaresova, J.; Cífková, R.; Sochor, O. Kardiovize brno 2030, a prospective cardiovascular health study in central Europe: Methods, baseline findings and future directions. Eur. J. Prev. Cardiol. 2017, 25, 54-64. [CrossRef] [PubMed]

18. Hruskova, J.; Maugeri, A.; Podroužková, H.; Štípalová, T.; Jakubík, J.; Barchitta, M.; Medina-Inojosa, J.R.; Homolka, M.; Agodi, A.; Kunzova, S.; et al. Association of cardiovascular health with epicardial adipose tissue and intima media thickness: The kardiovize study. J. Clin. Med. 2018, 7, 113. [CrossRef] [PubMed]

19. Maugeri, A.; Kunzova, S.; Medina-Inojosa, J.R.; Agodi, A.; Barchitta, M.; Homolka, M.; Kiacova, N.; Bauerova, H.; Sochor, O.; Lopez-Jimenez, F.; et al. Association between eating time interval and frequency with ideal cardiovascular health: Results from a random sample Czech urban population. Nutr. Metab. Cardiovasc. Dis. 2018. [CrossRef] [PubMed]

20. Harris, P.A.; Taylor, R.; Thielke, R.; Payne, J.; Gonzalez, N.; Conde, J.G. Research electronic data capture (redcap) - A metadata-driven methodology and workflow process for providing translational research informatics support. J. Biomed. Inform. 2009, 42, 377-381. [CrossRef] [PubMed]

21. Brussaard, J.H.; Löwik, M.R.; Steingrímsdóttir, L.; Møller, A.; Kearney, J.; De Henauw, S.; Becker, W.; Group, E. A European food consumption survey method-Conclusions and recommendations. Eur. J. Clin. Nutr. 2002, 56 (Suppl. 2), S89-S94. [CrossRef] [PubMed]

22. Ruprich, J.; Dofkova, M.; Rehurkova, I.; Slamenikova, E.; Resova, D. Individual Food Consumption-The National Study SISP04. CHFCH NIPH in Prague. Available online: http://www.chpr.szu.cz/ spotrebapotravin.htm (accessed on 1 April 2018). 
23. Willett, W.; Stampfer, M.J. Total energy intake: Implications for epidemiologic analyses. Am. J. Epidemiol. 1986, 124, 17-27. [CrossRef] [PubMed]

24. Craig, C.L.; Marshall, A.L.; Sjostrom, M.; Bauman, A.E.; Booth, M.L.; Ainsworth, B.E.; Pratt, M.; Ekelund, U.; Yngve, A.; Sallis, J.F.; et al. International physical activity questionnaire: 12-country reliability and validity. Med. Sci. Sports Exerc. 2003, 35, 1381-1395. [CrossRef] [PubMed]

25. Suliga, E.; Kozieł, D.; Cieśla, E.; Rębak, D.; Głuszek, S. Dietary patterns in relation to metabolic syndrome among adults in Poland: A cross-sectional study. Nutrients 2017, 9, 1366. [CrossRef] [PubMed]

26. Kimokoti, R.W.; Gona, P.; Zhu, L.; Newby, P.K.; Millen, B.E.; Brown, L.S.; D’Agostino, R.B.; Fung, T.T. Dietary patterns of women are associated with incident abdominal obesity but not metabolic syndrome. J. Nutr. 2012, 142, 1720-1727. [CrossRef] [PubMed]

27. Romaguera, D.; Ängquist, L.; Du, H.; Jakobsen, M.U.; Forouhi, N.G.; Halkjær, J.; Feskens, E.J.; van der A, D.L.; Masala, G.; Steffen, A.; et al. Food composition of the diet in relation to changes in waist circumference adjusted for body mass index. PLoS ONE 2011, 6, e23384. [CrossRef] [PubMed]

28. Barbaresko, J.; Siegert, S.; Koch, M.; Aits, I.; Lieb, W.; Nikolaus, S.; Laudes, M.; Jacobs, G.; Nöthlings, U. Comparison of two exploratory dietary patterns in association with the metabolic syndrome in a northern german population. Br. J. Nutr. 2014, 112, 1364-1372. [CrossRef] [PubMed]

29. Zhang, Q.; Chen, X.; Liu, Z.; Varma, D.S.; Wan, R.; Wan, Q.; Zhao, S. Dietary patterns in relation to general and central obesity among adults in southwest china. Int. J. Environ. Res. Public Health 2016, 13, 1080. [CrossRef] [PubMed]

30. Sadakane, A.; Tsutsumi, A.; Gotoh, T.; Ishikawa, S.; Ojima, T.; Kario, K.; Nakamura, Y.; Kayaba, K. Dietary patterns and levels of blood pressure and serum lipids in a Japanese population. J. Epidemiol. 2008, 18, 58-67. [CrossRef] [PubMed]

31. Shin, J.Y.; Kim, J.M.; Kim, Y. Associations between dietary patterns and hypertension among Korean adults: The Korean national health and nutrition examination survey (2008-2010). Nutr. Res. Pract. 2013, 7, $224-232$. [CrossRef] [PubMed]

32. Sun, J.; Buys, N.J.; Hills, A.P. Dietary pattern and its association with the prevalence of obesity, hypertension and other cardiovascular risk factors among Chinese older adults. Int. J. Environ. Res. Public Health 2014, 11, 3956-3971. [CrossRef] [PubMed]

33. O'Connor, L.E.; Kim, J.E.; Campbell, W.W. Total red meat intake of $\geq 0.5$ servings/d does not negatively influence cardiovascular disease risk factors: A systemically searched meta-analysis of randomized controlled trials. Am. J. Clin. Nutr. 2017, 105, 57-69. [CrossRef] [PubMed]

34. Jia, Q.; Xia, Y.; Zhang, Q.; Wu, H.; Du, H.; Liu, L.; Wang, C.; Shi, H.; Guo, X.; Liu, X.; et al. Dietary patterns are associated with prevalence of fatty liver disease in adults. Eur. J. Clin. Nutr. 2015, 69, 914-921. [CrossRef] [PubMed]

35. Yang, C.Q.; Shu, L.; Wang, S.; Wang, J.J.; Zhou, Y.; Xuan, Y.J.; Wang, S.F. Dietary patterns modulate the risk of non-alcoholic fatty liver disease in Chinese adults. Nutrients 2015, 7, 4778-4791. [CrossRef] [PubMed]

36. Beck, K.L.; Jones, B.; Ullah, I.; McNaughton, S.A.; Haslett, S.J.; Stonehouse, W. Associations between dietary patterns, socio-demographic factors and anthropometric measurements in adult New Zealanders: An analysis of data from the 2008/09 New Zealand adult nutrition survey. Eur. J. Nutr. 2018, 57, 1421-1433. [CrossRef] [PubMed]

37. Mazidi, M.; Kengne, A.P. Nutrient patterns and their relationship with general and central obesity in US adults. Eur. J. Clin. Investig. 2017. [CrossRef] [PubMed]

38. Rezagholizadeh, F.; Djafarian, K.; Khosravi, S.; Shab-Bidar, S. A posteriori healthy dietary patterns may decrease the risk of central obesity: Findings from a systematic review and meta-analysis. Nutr. Res. 2017, 41,1-13. [CrossRef] [PubMed]

39. Zhang, M.; Zhu, Y.; Li, P.; Chang, H.; Wang, X.; Liu, W.; Zhang, Y.; Huang, G. Associations between dietary patterns and impaired fasting glucose in Chinese men: A cross-sectional study. Nutrients 2015, 7, 8072-8089. [CrossRef] [PubMed]

40. Villegas, R.; Salim, A.; Flynn, A.; Perry, I.J. Prudent diet and the risk of insulin resistance. Nutr. Metab. Cardiovasc. Dis. 2004, 14, 334-343. [CrossRef]

41. Montonen, J.; Knekt, P.; Härkänen, T.; Järvinen, R.; Heliövaara, M.; Aromaa, A.; Reunanen, A. Dietary patterns and the incidence of type 2 diabetes. Am. J. Epidemiol. 2005, 161, 219-227. [CrossRef] [PubMed] 
42. Adriano, L.S.; Sampaio, H.A.; Arruda, S.P.; Portela, C.L.; de Melo, M.L.P.; Carioca, A.A.; Soares, N.T. Healthy dietary pattern is inversely associated with non-alcoholic fatty liver disease in elderly. Br. J. Nutr. 2016, 115, 2189-2195. [CrossRef] [PubMed]

43. Barchitta, M.; Maugeri, A.; Quattrocchi, A.; Agrifoglio, O.; Scalisi, A.; Agodi, A. The association of dietary patterns with high-risk human papillomavirus infection and cervical cancer: A cross-sectional study in Italy. Nutrients 2018, 10, 469. [CrossRef] [PubMed]

44. Wagner, A.; Dallongeville, J.; Haas, B.; Ruidavets, J.B.; Amouyel, P.; Ferrières, J.; Simon, C.; Arveiler, D. Sedentary behaviour, physical activity and dietary patterns are independently associated with the metabolic syndrome. Diabetes Metab. 2012, 38, 428-435. [CrossRef] [PubMed]

2018 by the authors. Licensee MDPI, Basel, Switzerland. This article is an open access article distributed under the terms and conditions of the Creative Commons Attribution (CC BY) license (http://creativecommons.org/licenses/by/4.0/). 